\title{
A 61-year-old female patient with right-sided pleuritic chest pain and fatigue
}

\author{
N. Messaoudi*, J. De Cocker*, G. Van den Eynden" ${ }^{\#}$ A. Janssens ${ }^{\star}$, R.A. Salgado ${ }^{+}$, \\ E.A. Van Marck ${ }^{\#}$ and P.E.Y. Van Schil*
}

\section{CASE REPORT}

A 61-yr-old Caucasian female patient was investigated because of right-sided pleuritic chest pain. The patient had a 2-month history of chronic, nonproductive cough, extreme fatigue, night sweats and reduced exercise tolerance. Initially, the patient was treated with antibiotics for presumed pneumonia. She had smoked for $>20$ pack-yrs and her medical history included parotidectomy for Warthin tumour, resection of a benign cyst in the left breast and varicectomy.

Physical examination showed no clinical signs of respiratory distress. Sinus rhythm was present with a heart rate of $88 \mathrm{bpm}$ and blood pressure of 135/80 mmHg. Cardiopulmonary auscultation revealed no significant abnormalities and there was no hepatomegaly or limb oedema. No lymphadenopathy was found in the neck, supraclavicular, axillary or inguinal regions.

The laboratory results showed an elevated C-reactive protein of $5.02 \mathrm{mg} \cdot \mathrm{dL}^{-1}$. Low haemoglobin and haematocrit values were in keeping with anaemia. Studies of coagulability were all within normal ranges. Arterial blood gas analysis while breathing ambient air showed an arterial oxygen tension of $66 \mathrm{~mm} \mathrm{Hg}$, carbon dioxide arterial tension of $40 \mathrm{mmHg}$, $\mathrm{pH}$ of 7.43 and arterial oxygen saturation of $94 \%$. Pulmonary function tests revealed a mild restrictive defect with a reduced diffusing capacity.
A chest radiograph (fig. 1) and computed tomography (CT) scans (figs 2 and 3) were performed and the patient underwent bronchoscopy and mediastinoscopy. Bronchoscopy revealed no endobronchial involvement. Subsequent biopsies were all found to be negative.

All accessible lymph nodes in the superior mediastinum and the region of the right mainstem bronchus were biopsied by cervical mediastinoscopy. Extensive unilateral mediastinal fibrosis was present around the right pulmonary artery and mainstem bronchus.

Subsequently, the patient underwent thoracoscopy to obtain a more precise diagnosis. However, extensive inflammation and multiple dense adhesions rendered appropriate investigation impossible, even after opening of the pericardium. Conversion to a limited thoracotomy was performed. Operative findings were general hepatisation of the right lung and nodular lesions in the right lower lobe, which were resected by wedge excision. Central biopsies were also taken.

Despite these investigations, a final diagnosis could not be obtained. As the right lung was afunctional, an intrapericardial pneumonectomy was performed and definitive pathological examination revealed the final diagnosis (figs 4 and 5).

Depts of *Thoracic and Vascular Surgery, "Pathology, "Pulmonary Medicine, and +Radiology, Antwerp University Hospital, Edegem, Belgium. STATEMENT OF INTEREST: None declared. 


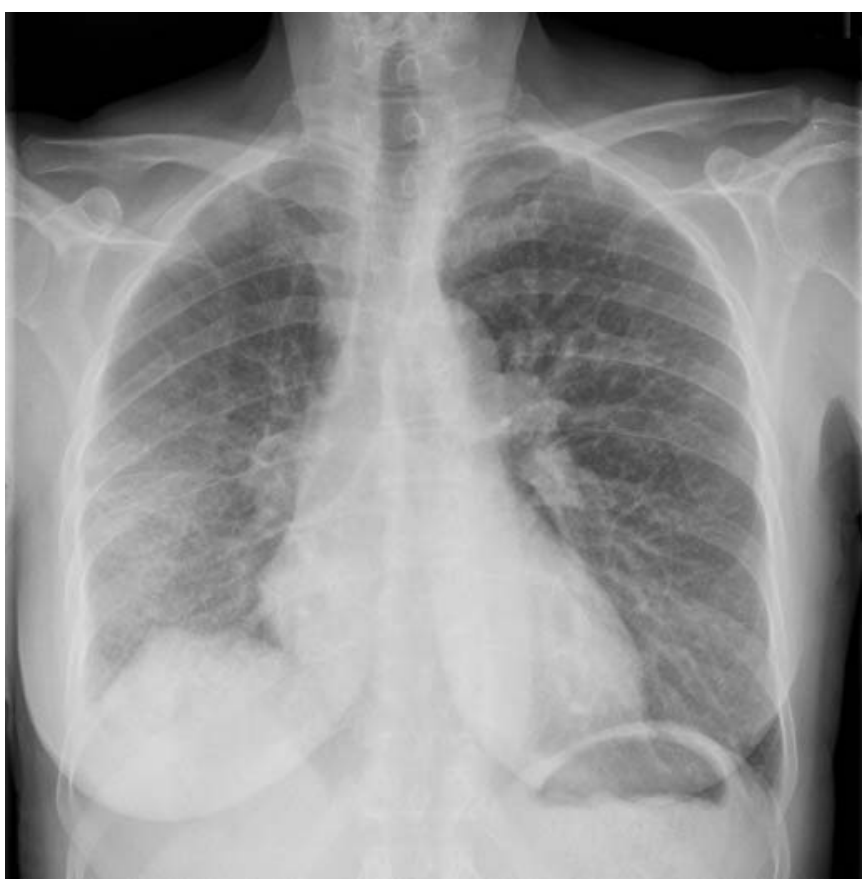

FIGURE 1. Chest radiograph.
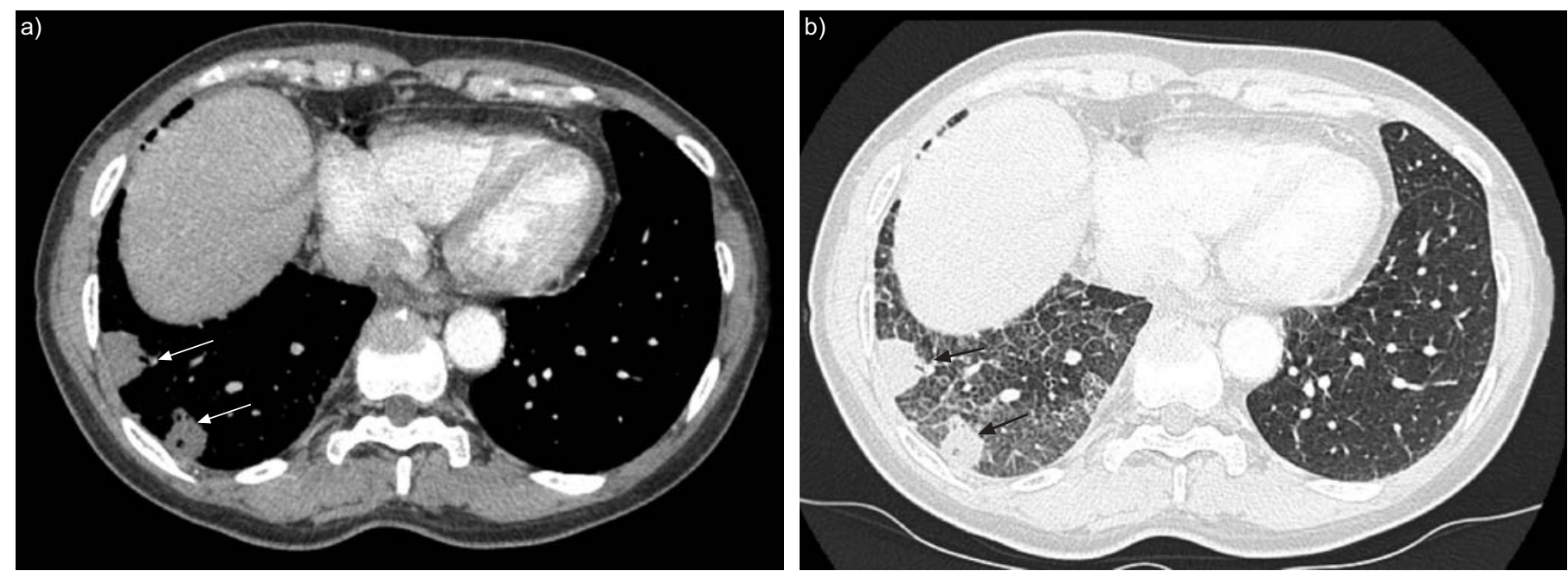

FIGURE 3. Axial contrast-enhanced chest computed tomography scan. a) Mediastinal window and b) lung window. Two peripherally located wedge-shaped focal consolidations areas at the right lung base are indicated by the arrows.

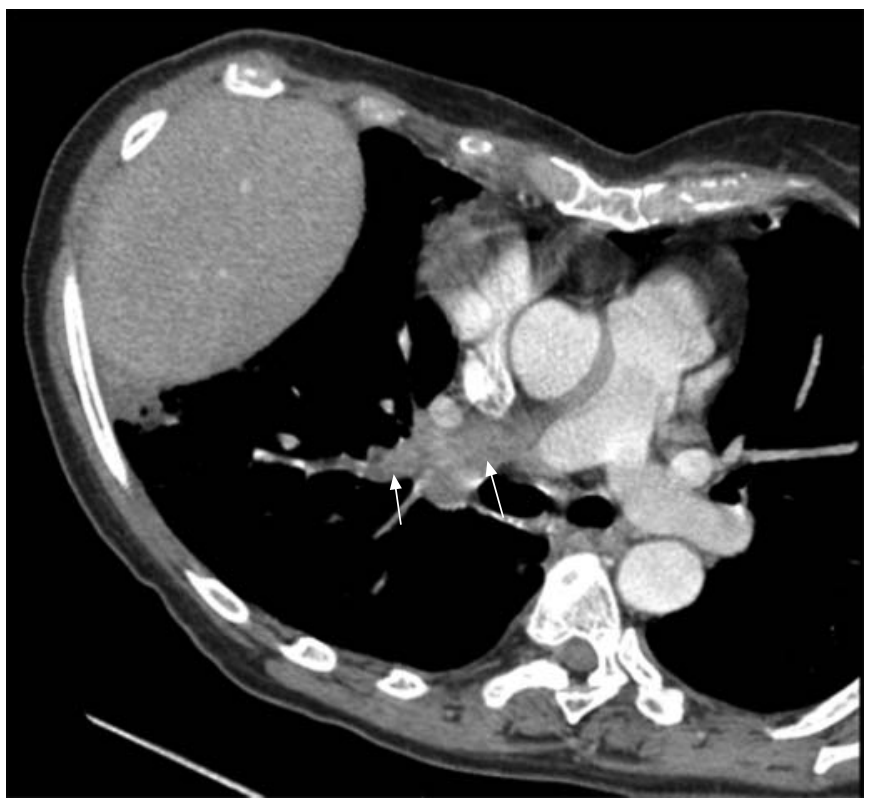

FIGURE 2. Axial contrast-enhanced chest computed tomography scan Extensive soft tissue mass in the right pulmonary artery, extending in different side branches, is indicated by the arrows. 

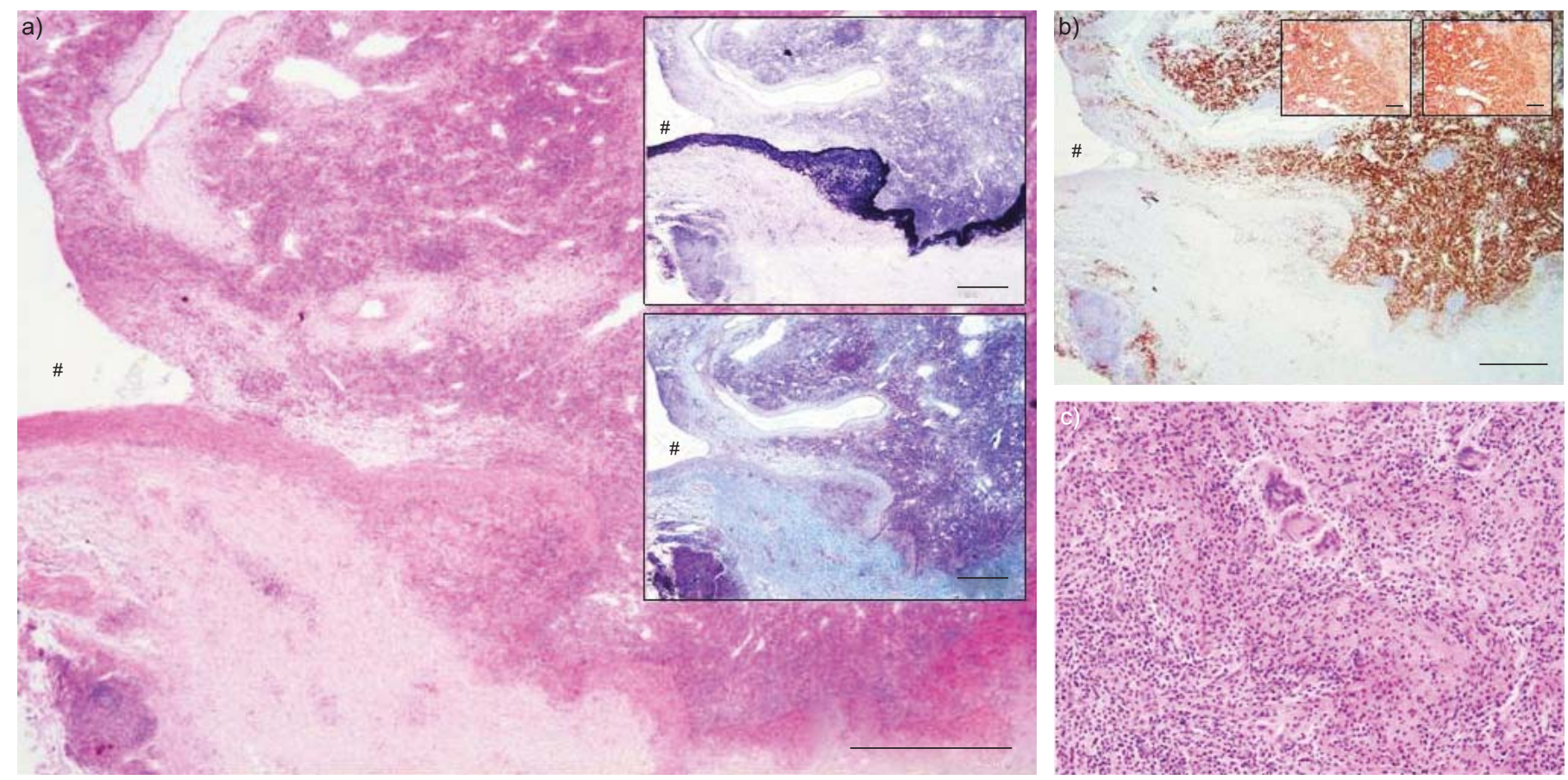

FIGURE 4. Histopathological examination of lung biopsies. a) Histopathological examination of the pulmonary artery wall. The upper (Von Giesson's elastic stain) and lower (Masson's trichrome stain) insets show the membrane elastica interna of the pulmonary artery and the extensive fibrosis, respectively. b) The inflammatory infiltrate is mainly composed of CD138 immunoreactive plasma cells. The insets show that this population is polyclonal (immunohistochemical stain for $\kappa$ and $\lambda$ light chain immunoglobulins, left and right insets, respectively). c) The presence of many giant cells was also noted. ${ }^{*}$ : the fibro-inflammatory process which completely occludes the lumen. Scale bars $=1,000 \mu \mathrm{m}$.
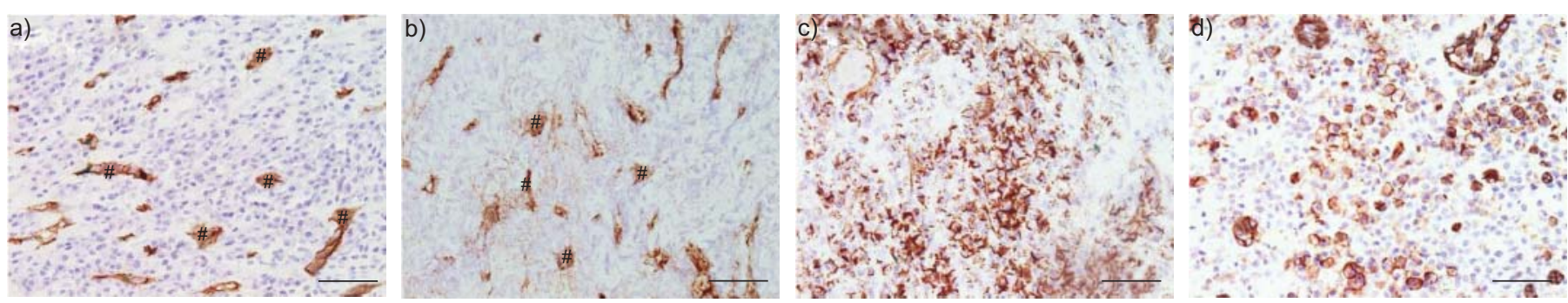

FIGURE 5. Histopathological examination of the endothelial markers in $\mathrm{a}-\mathrm{c}$ ) the patient and d) plasma cell rich granulation tissue. a) CD34, b) Factor VIII, and c and d) CD31. \#: endothelial immunoreactivity of the blood vessels. Scale bars $=50 \mu \mathrm{m}$.

\section{BEFORE TURNING THE PAGE, INTERPRET THE CHEST RADIOGRAPH, CT SCANS AND LUNG BIOPSY PATHOLOGY AND SUGGEST A DIAGNOSIS.}




\section{INTERPRETATION}

\section{Radiology}

The chest radiograph (fig. 1) shows elevation of the right dome of the diaphragm with diffuse ground-glass attenuation in the right lung base. Furthermore, there is superimposed intralobular and interlobular septal thickening.

\section{Computed tomography}

A curved multiplanar reformatted contrast-enhanced CT image along the course of the right pulmonary artery and side branches is shown in figure 2. An extensive soft tissue mass is observed in the right pulmonary artery, extending in different side branches (fig. 2); in this way almost completely occluding the pulmonary arterial supply of the right lung. Enlarged mediastinal and hilar lymph nodes are also present (not shown).

Figure 3 shows axial contrast-enhanced CT images at a lower level and reveals two peripherally located wedge-shaped focal consolidations areas (fig. 3) at the right lung base surrounded by intralobular and interlobular septal thickening. These findings are consistent with extensive lung infarction.

\section{Pathology}

Gross examination of the pneumonectomy specimen shows a white/gray mass in the wall of the pulmonary artery that (sub)totally occludes the lumen over $1.7 \mathrm{~cm}$. The process also expands to the periarterial connective tissue. The pulmonary veins and right bronchial tree show no abnormalities. Furthermore, there are triangular, subpleural infarctions in all three lobes of the right lung.

Histopathological examination demonstrates that all layers of the pulmonary artery wall are involved in a fibro-inflammatory process, which completely occludes the lumen. The membrane elastica interna of the pulmonary artery and the extensive fibrosis is shown in figure $4 \mathrm{~b}$. In addition, the inflammatory infiltrate is mainly composed of CD138 immunoreactive plasma cells (fig. 4b) and shows that this population is polyclonal. The presence of many giant cells was also noted (fig. 4c).

Immunohistochemical stains for endothelial cell markers (CD34, CD31 and Factor VIII) show reactivity of blood vessels for all three markers (fig. 5). The abundant plasma cells in the lesion show reactivity for CD31 but not for CD34 or Factor VIII. However, there are no atypical CD34, CD31 or Factor VIII immunoreactive cells. The CD31 stain of plasma cell rich granulation tissue at the border of an abscess demonstrates the CD31 immunoreactivity of reactive plasma cells.

Diagnosis: Inflammatory pseudotumour causing right pulmonary artery occlusion with subsequent infarction of the lung.

\section{Clinical course}

Given the complete occlusion of the right pulmonary artery and the fact that the right lung was infarcted and afunctional, a right pneumonectomy was carried out with the patient on cardiopulmonary bypass, which allowed transection of the right pulmonary artery at its origin from the pulmonary trunk. The patient made an uneventful post-operative recovery. Follow-up 2 yrs after surgery showed a normal status after right pneumonectomy without signs of recurrent or metastatic disease.

\section{DISCUSSION}

Inflammatory pseudotumour is considered to be a rare, benign, neoplastic soft-tissue tumour of unknown origin; its nomenclature is inconsistent. Plasma cell granuloma, inflammatory myofibroblastic tumour, histiocytoma, xanthoma, fibroxanthoma, xanthogranuloma, plasma cell tumour, fibrous xanthoma or xanthomatous pseudotumour are similar terms depending on the predominant cellular components. As a result, little is known about its true incidence. However, an incidence of $0.04-1.2 \%$ of all resected tumours of the lung has been reported [1].

A solitary pulmonary nodule is often the characteristic presentation of an inflammatory pseudotumour. Extraparenchymal localisation, including mediastinal invasion and pulmonary artery lumen involvement, is a rare occurrence [2, 3] and patients of all ages can be affected. Nonetheless, more than half of patients are aged $<40 \mathrm{yrs}$ at the time of diagnosis [3]. Symptoms are nonspecific and include cough, haemoptysis, chest pain, dyspnoea, fatigue, fever and clubbing. Involvement of mediastinal structures mimics neoplasia.

No particular findings on laboratory examination exist since elevated inflammatory parameters are nonspecific. Radiological investigation only confirms the presence of a solitary pulmonary lesion. Minimally invasive procedures are often insufficient to provide the diagnosis of inflammatory pseudotumours, as demonstrated in the present case. Bronchoscopy and cytological examinations are often negative. Percutaneous needle biopsy may induce a diagnostic dilemma because of the difficulty to differentiate inflammatory pseudotumour from fibrohistiocytic neoplasms, nodular sclerosing Hodgkin's lymphoma, primary lung cancer and especially fibrosing mediastinitis [4]. In addition, due to the specific unilateral localisation of the lesion in the present case, fibrosing mediastinitis is less probable.

The main differential diagnosis is an angiosarcoma. Derived from endothelial cells, this relatively uncommon malignant tumour most commonly presents in the lung. Radiologically, angiosarcoma mimics the appearance as seen in the present case. Therefore, immunohistochemical staining with endothelial markers CD34, CD31 and Factor VIII was performed to exclude an angiosarcoma of the right pulmonary artery. As shown in figure 5, endothelial immunoreactivity of the blood vessels was confirmed by the three markers. CD31 was also positive in staining the CD138 immunoreactive plasma cells, but CD34 and Factor VIII were negative. Positive immunohistochemical staining for CD31 has previously been reported in reactive extramedullar plasma cells [4]. This was demonstrated in the present study in the plasma cell rich granulation tissue (fig. 5d). Most importantly, there were no atypical cells with endothelial differentiation, thus making angiosarcoma less probable. An uneventful post-operative follow-up of the patient also contradicts an angiosarcoma.

The present case was unusual as the patient presented with right pulmonary artery obstruction caused by intraluminal invasion of the inflammatory pseudotumour and adjacent fibrosis. Thus, pulmonary vasculitis was considered in the differential diagnosis. Slow progressive growth of the lesion, concurrent arterial occlusion and small emboli finally gave rise to pulmonary infarction.

Surgical resection has both diagnostic and therapeutic value. The recommended surgical approach is complete resection to 
enable excellent prognosis and survival $[5,6]$. For inoperable cases, nonsurgical treatment options including radiotherapy, chemotherapy and steroids have been attempted with less encouraging results [7-10]. Spontaneous regression is very rare.

Although the most common presentation of inflammatory pseudotumour is a solitary pulmonary nodule, mediastinal localisation can be observed. Using minimally invasive methods, an accurate histopathological diagnosis remains difficult to obtain. In order to make a definitive diagnosis, exploratory surgery is suggested, with complete resection being the treatment of choice.

\section{REFERENCES}

1 Melloni G, Carretta A, Ciriaco P, et al. Inflammatory pseudotumor of the lung in adults. Ann Thorac Surg 2005; 79: 426-432.

2 Warter A, Satge D, Roeslin N. Angioinvasive plasma cell granulomas of the lung. Cancer 1987; 59: 435-443.

3 Agrons GA, Rosado-de-Christenson ML, Kirejczyk WM, Conran RM, Stocker JT. Pulmonary inflammatory pseudotumor: radiologic features. Radiology 1998; 206: 511-518.
4 Govender D, Harilal P, Dada M, Chetty R. CD31 (JC70) expression in plasma cells: an immunohistochemical analysis of reactive and neoplastic plasma cells. J Clin Pathol 1997; 50: 490-493.

5 Cerfolio RJ, Allen MS, Nascimento AG, et al. Inflammatory pseudotumors of the lung. Ann Thorac Surg 1999; 67: 933-936.

6 Copin MC, Gosselin BH, Ribet ME. Plasma cell granuloma of the lung: difficulties in diagnosis and prognosis. Ann Thorac Surg 1996; 61: 1477-1482.

7 Imperato JP, Folkman J, Sagerman RH, Cassady JR. Treatment of plasma cell granuloma of the lung with radiation therapy. A report of two cases and a review of the literature. Cancer 1986; 57: 2127-2129.

8 Bahadori M, Liebow AA. Plasma cell granulomas of the lung. Cancer 1973; 31: 191-208.

9 Boman F, Champigneulle J, Boccon-Gibod L, et al. Inflammatory myofibroblastic tumor of the lung with endobronchial, infiltrating, multifocal and recurrent form. Ann Pathol 1995; 15: 207-210.

10 Shirakusa T, Kusano T, Motonaga R, Eimoto T. Plasma cell granuloma of the lung-resection and steroid therapy. Thorac Cardiovasc Surg 1987; 35: 185-188. 